\title{
Innovación docente y participación ciudadana en proyectos de regeneración urbana. PIME "Desis Lab: Proyecto parques urbanos de barrio”
}

\author{
Ana Portalés Mañanós ${ }^{a}$, David Urios Mondéjar ${ }^{b}$, Juan Colomer Alcácer ${ }^{c}$, Maite \\ Palomares Figueres $^{d}$, Consuelo Esteve Sendra ${ }^{e}$ y Asenet Sosa Espinosa $^{f}$
}

a'Departamento de Urbanismo, anporma@urb.upv.es, ${ }^{\mathrm{b}}$ Departamento de Urbanismo, umdav@urb.upv.es. ${ }^{\mathrm{c} D e p a r t a m e n t o ~ d e ~ U r b a n i s m o, ~ j u a n c o l o m e r a l c a c e r @ g m a i l . c o m . ~}{ }^{\mathrm{d}}$ Departamento de Composición Arquitectónica, mapafi@cpa.upv.es, ${ }^{\mathrm{e}}$ Departamento de Dibujo cheleesteve00@gmail.com, y ${ }^{\mathrm{f}}$ Departamento de Urbanismo, assoes@urb.upv.es.

\begin{abstract}
A PIME teaching innovation project has been presented (Project of Improvement and innovation in education). It is based on improvement and innovation in education. It is an urban regeneration project of multidisciplinary nature which is currently under development. Teachers and students of different qualifications (architecture, urban planning, design, sociology and botany) take an active part contributing to the development of an overall plan. The PIME is framed within the following methodogies: $A B P$ (Project-based learning:) and APS (Servicelearning). This teaching innovation succeeded in creating a teacher Web platform for citizen participation (www.valenciaparcsdebarri.es)
\end{abstract}

keywords: service learning, project-based learning, citizen participation, urban regeneration, multidisciplinary project.

\section{Resumen}

Se presenta un proyecto de innovación docente PIME (Proyecto de Innovación y Mejora Educativa) basado en el empleo de metodologías docentes activas aplicadas a la enseñanza de una asignatura de carácter proyectual que trata temas de regeneración urbana. Este proyecto de innovación tiene un carácter multidisciplinar y actualmente se encuentra en fase de desarrollo. Participan profesores, alumnos y alumnas de distintas titulaciones (arquitectura, urbanismo, diseño, sociología y botánica) contribuyendo en el desarrollo de un proyecto global. El PIME, que cuenta entre sus antecedentes con la creación de una plataforma web docente y de participación ciudadana www.valenciaparcsdebarri.es, se enmarca dentro de las metodologías APP (Aprendizaje basado en Proyectos), y APS (Aprendizaje Servicio).

Palabras clave: aprendizaje servicio, aprendizaje basado en proyectos, participación ciudadana, regeneración urbana, proyecto multidisciplinar. 


\section{Introducción}

En la comunicación se presenta un Proyecto de Innovación y Mejora Educativa (PIME) basado en el empleo de metodologías docentes activas aplicadas a la enseñanza de asignaturas de carácter proyectual. Esta innovación se plantea en la asignatura Urbanística I de $2^{\circ}$ curso del Grado en Fundamentos de Arquitectura en la que se desarrolla un proyecto de regeneración urbana.

Coincidiendo con la reflexión: "Cuando se piensa en la regeneración urbana, se acostumbra a enfatizar la necesidad de las visiones integradoras. En los últimos años, eso significa considerar cada vez más los factores sociales, económicos y ambientales, además de físicos y constructivos" (Díez-Medina, C., \& Monclús-Fraga, J., 2017) planteamos la necesidad de establecer alianzas entre la ciudad y ciudadanía, así como con las distintas disciplinas que gravitan en torno al espacio público.

Estas conexiones entre ciudad y disciplinas relacionadas con el proyecto urbano, se pretenden trasladar al ámbito docente a través del PIME que se presenta. Así, como objetivos principales de la innovación docente señalamos por un lado, e introducir la multidisciplinareidad que caracteriza el trabajo profesional del arquitecto en el campo del urbanismo, y por otro, acercar al alumnado a la resolución de temas reales que les aproximen a las condiciones en las que podrán desarrollar su trabajo profesional.

La colaboración en un proyecto de disciplinas afines y complementarias al urbanismo, y el acercamiento a la realidad urbana, aumenta la motivación del alumnado y supone una mejora en los resultados docentes de los proyectos.

Otros pilares de apoyo en el desarrollo de este proyecto son la apertura de la Universidad a la sociedad a través del Aprendizaje Servicio (APS), la participación ciudadana y el desarrollo de trabajo en equipo. Generalmente en los proyectos de regeneración urbana de espacios públicos es habitual el trabajo en equipos multidisciplinares (arquitectos, ingenieros, sociólogos, diseñadores etc.). Este modo de trabajar se traslada a las aulas en el marco de una asignatura con una metodología de Aprendizaje Basado en Proyectos ( $A B P$ ). Así también, en este proyecto, se valora la importancia de transmitir el conocimiento e interaccionar con la sociedad, por ello consideramos necesario la apertura de la universidad a la ciudadanía. Mediante la interacción con los ciudadanos a través de una página web, obteniendo una información relevante para el desarrollo del proyecto.

El PIME se plantea partiendo de experiencias docentes previas en las que se han obtenido como resultados, entre otros, la creación de una página web de participación ciudadana www.valenciaparcsdebarri.es en la que han colaborado más de 300 alumnos y alumnas de la UPV. También cabría destacar el plan de difusion previsto para los resultados del PIME, a través de la incorporación de este proyecto dentro de la red Desis Lab.

(cc) EY-NC-ND 2018, Universitat Politècnica de València

Congreso IN-RED (2018) 


\section{Objetivos}

Partiendo de la reflexión anterior, en el PIME se plantean una serie de objetivos generales y específicos que se desarrollan a continuación

\subsection{Generales}

Incorporar a la docencia la interdisciplinareidad necesaria para mejorar el resultado de un proyecto docente en el que la temática es la regeneración urbana y la sostenibilidad en el espacio público. Para ello se impartirán clases magistrales de disciplinas complementarias a la urbanística, como la sociología o la botánica, así como clases técnicas de iluminación y accesibilidad en el espacio público.

Abrir la Universidad a la sociedad, a la ciudadanía y a las instituciones mediante la aplicación de la metodología Aprendizaje Servicio, de modo que el trabajo desarrollado por el alumnado, por un lado responda a las necesidades sociales reales y por otro tenga una utilidad para la administración pública. En este caso a través de un convenio de colaboración con l'Ajuntament d’Algemesí.

Utilizar la experiencia docente como laboratorio de diseño donde se inicien nuevos proyectos. Si un movimiento mundial hacia la sostenibilidad exige el mejor uso posible de los recursos existentes, las escuelas implicadas en el PIME, con todo su potencial en términos de entusiasmo del alumnado participante, deben considerarse como un recurso social muy prometedor: un potencialmente poderoso promotor del cambio sostenible.

Dar visibilidad a los resultados obtenidos del PIME desde los Desis Lab. Utilizar las Escuelas de Diseño, Arquitectura, Bellas Artes, Agrónomos como agentes del cambio y la web www.desislabspain.webs.upv.es para la difusión de resultados.

\subsection{Específicos}

Coordinación y desarrollo de trabajos transversales e interdisciplinares en relación a los proyectos de regeneración urbana. La incorporación de disciplinas complementarias y clases técnicas especificas ontribuye a la mejora proyectual, social y ambiental de estos espacios públicos.

Aplicar y trabajar en la docencia la competencia número 7: "Responsabilidad ética, medioambiental y profesional”, ya que el alumnado trabaja con temas que le acercan a la realidad social y medioambiental, a través del proyecto de mejora del espacio público.

Continuar y perfeccionar las metodologías docentes y de participación ciudadana iniciadas con la plataforma web www.valenciaparcsdebarri.es como antecedente a esta innovación.

Potenciar el trabajo en equipo y las aportaciones interdisciplinares.

Aportar conocimiento, experiencias e iniciativas a la red Internacional DESIS LAB

Difundir las metodologías y los resultados docentes de la UPV, a la sociedad y en el ámbito internacional a través de la red para aumentar la sinergia mediante el desarrollo de marcos y 
plataformas para conectar los diversos casos locales en los proyectos regionales más grandes.

\section{Desarrollo de la innovación}

\subsection{Antecedentes}

\subsubsection{Experiencias previas}

Como experiencias previas cabría exponer, a parte de la aplicación de la metodología ABP y APS en las ciudades de Valencia y Cullera durantes los cursos 2015-16 y 2016-17, la creación de la plataforma web valenciaparcsdebarri.es. Esta herramienta web en la que han participado alrededor de 300 alumnos y alumnas durante los cursos 2014-15 y 2015-16 surgió de la preocupación por la mejora en la toma de decisiones proyectuales en la regeneración urbana de los espacios públicos. Con el fin de ahondar en el programa de necesidades del espacio público, esta web permite obtener información de los usuarios y analizar las variables que inciden en el grado de uso y de confort de los usuarios, como calidad ambiental, accesibilidad, diseño y materialidad, entre otros.

Se trata de una plataforma web de investigación docente social y urbana que facilita el intercambio de información y colaboración entre los usuarios de los parques, el alumnado, el profesorado y los organismos públicos. en torno a los parques de barrio de Valencia. La web incluye la localización y datos básicos de los parques de barrio de Valencia y supone una ventana abierta a los usuarios del espacio público para que conozcan opinen y valoren los parques de barrio, y de este modo, favorecer la mejora proyectual de estos espacios libres.

\subsubsection{Pertenencia y colaboración del equipo con la red DESIS_Lab}

Entre los profesores integrantes del equipo que desarrolla el PIME, cabe destacar la pertenencia y participación de algunos en la red DESIS-Lab. Esta asociación sin ánimo de lucro y de carácter cultural tiene como propósito promover el diseño para la innovación social en las instituciones de educación superior, donde a través de un diseño, pueda generar conocimiento útil para crear cambios sociales significativos. Desde este punto de vista, la capacidad de difusion que brinda la red para incorporar la experiencia del PIME, será aprovechada para dar visibilidad a nivel internacional de la experiencia.

Durante la última década la innovación social se ha extendido a una gran variedad de actores sociales en todo el mundo (instituciones, empresas, organizaciones sin ánimo de lucro y, sobre todo, las redes de personas en colaboración) que se han desplazado fuera de los modelos convencionales de pensar y hacer, generando una variedad iniciativas de prometedoras que proponen soluciones viables a problemas complejos como la regeneración urbana, la accesibilidad y la gestión de la energía.

La red DESIS posee Labs en todos los continentes. Hoy en día, la innovación social está generando una constelación de pequeñas iniciativas. Sin embargo, si se crean condiciones favorables, estas pequeñas invenciones sociales, locales y sus prototipos del trabajo

(cc) EY-NC-ND 2018, Universitat Politècnica de València

Congreso IN-RED (2018) 
resultante pueden propagar para generar cambios sostenibles y sociales a gran escala. (Fig.

1)

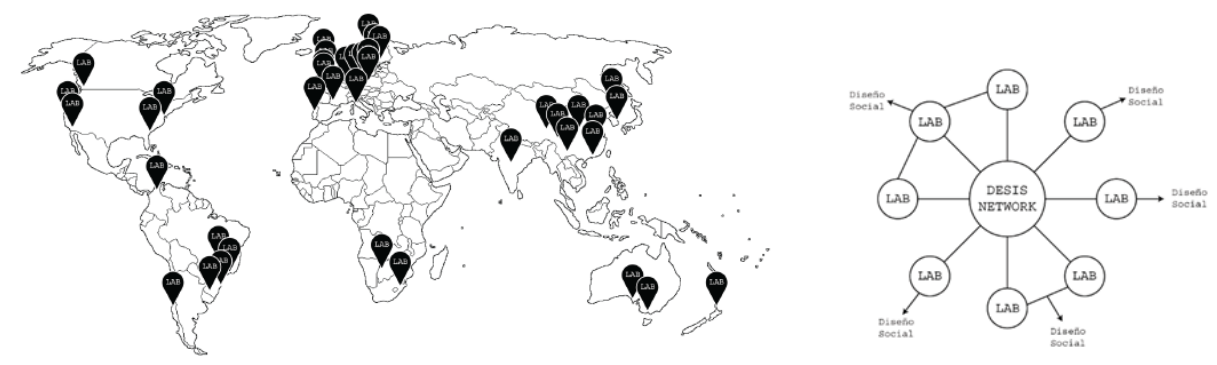

Fig. 1 Localización y esquema organizativo de la red DESIS-Lab

Actualmente el único Lab de DESIS Network en territorio español está en la ETSID/UPV. Desde la ETSA/UPV se ha solicitado el ingreso en dicha Red. El diseño para la innovación social puede encontrar en la ETSA una experiencia piloto.

\subsection{Contenido y alcance de la innovación}

El proyecto se desarrollará mayoritariamente en la asignatura Urbanística I del Grado en Fundamentos de Arquitectura en la que se realiza un trabajo docente consistente en un proyecto de regeneración urbana en parques de barrio de Valencia. El resto de asignaturas implicadas en el PIME, participarán de forma parcial, mediante la aportación de conferencias impartidas por el profesorado y trabajos específicos realizados por alumnos y alumnas que complementen a los desarrollados en la asignatura de Urbanismo. En esta asignatura (Urbanística I) se reúnen las condiciones para implementar la innovación docente, puesto que esta aportación multidisciplinar, redundará en la mejora del desarrollo de proyectos de regeneración urbana. Además, los resultados docentes de todas las asignaturas implicadas contribuirán, entre otros, a actualizar la documentación de la plataforma, y este modo abrir la Universidad a la sociedad y generar un efecto de retroalimentación entre los usuarios de la web y el alumnado.

Así en la asignatura Urbanística I, los alumnos y alumnas aprenden a conocer y a leer la ciudad en las distintas escalas. Inicialmente analizan la evolución urbana que caracteriza a las ciudades, en segundo lugar se produce un acercamiento a la escala del "barrio", como unidad morfológica y funcional dentro de las ciudades, y finalmente se desciende a la escala humana del espacio público. Es en esta escala en la que el alumnado desarrolla un proyecto urbano de mejora del espacio público, generalmente, en los parques urbanos de barrio. Así se les propone realizar un proyecto sobre estos espacios libres, públicos, de uso cotidiano, reducido tamaño y presentes en toda la ciudad.

El Proyecto de mejora de espacio público en el que trabajan los alumnos y alumnas se desarrolla este curso (2017-18) en el municipio de Algemesí. 


\subsection{Metodologías docentes aplicadas}

El Proyecto de innovación docente se basa en la aplicación de dos metodologías activas de la enseñanza, dirigidas a aproximar el trabajo que desarrolla el alumnado a la realidad profesional. Por un lado, se potencia la necesidad de realizar trabajo multidisciplinar y en equipo por medio de la metodología de Aprendizaje Basado en Proyectos (ABP). Por otro lado, se valora la importancia de trabajar en temas y contextos reales, abriendo la Universidad a la sociedad, desde la aplicación de la metodología Aprendizaje Servicio (APS).

\subsubsection{Aprendizaje basado en proyectos $(A B P)$}

Se trata de una metodología docente activa que organiza el proceso de enseñanza y aprendizaje a través de la elaboración de proyectos por parte de los alumnos y alumnas (Gülbahar y Tinmaz, 2006). El proceso de aprendizaje se produce de forma gradual mediante la implicación de los estudiantes en el desarrollo de un Proyecto, en este caso un Proyecto de mejora de un espacio público. Los estudiantes trabajan de manera activa, planificando, implementando y controlando proyectos que tienen una aplicación en el mundo real, yendo más allá del aula de la clase (Martí, 2010).

\subsubsection{Aprendizaje servicio}

El Aprendizaje Servicio (APS) implica una propuesta educativa que combina procesos de aprendizaje y de servicio a la comunidad en un solo proyecto bien articulado, en el que los participantes se forman al trabajar sobre necesidades reales del entorno con el objetivo de mejorarlo. Por su parte, para (Martínez-Odría, 2007) el APS es un método de enseñanza que enfatiza tanto el aprendizaje académico que se desarrolla en el aula, como la realización de un servicio voluntario a favor de las necesidades detectadas en la comunidad próxima.

Coincidiendo en buena parte con las definiciones anteriores, en nuestro caso el alumnado a través de sus trabajos prestan un servicio a la sociedad trabajando en temas y necesidades reales. Así se establece como emplazamiento de trabajo para los proyectos de regeneración urbana la población de Algemesí, existiendo un compromiso con el Ayuntamiento y con la ciudadanía de aportar ideas que puedan orientar a la administración en la toma de decisiones encaminadas a la resolución de problemas y necesidades de la ciudadanía en la regeneración urbana del espacio público.

\subsection{Fases del desarrollo de la innovación}

Septiembre 2017. Con el inicio de curso se desarrolla un seminario monográfico de evolución urbana de las ciudades con objeto de introducir al alumnado en la identificación de distintos tejidos urbanos. El resultado de este curso es de aplicación para la primera fase del trabajo que consiste en entender la ciudad como un conjunto, en nuestro caso Algemesí.

Octubre 2017. El mes de octubre, coincidiendo con la Semana de la Arquitectura del municipio, el Ayuntamiento de Algemesí recibió a nuestro alumnado en una Jornada en la 
que además, se realizó una visita de campo por toda la ciudad con la finalidad de obtener datos de los problemas y oportunidades detectados en el espacio público. (Fig.2/ Fig.3)
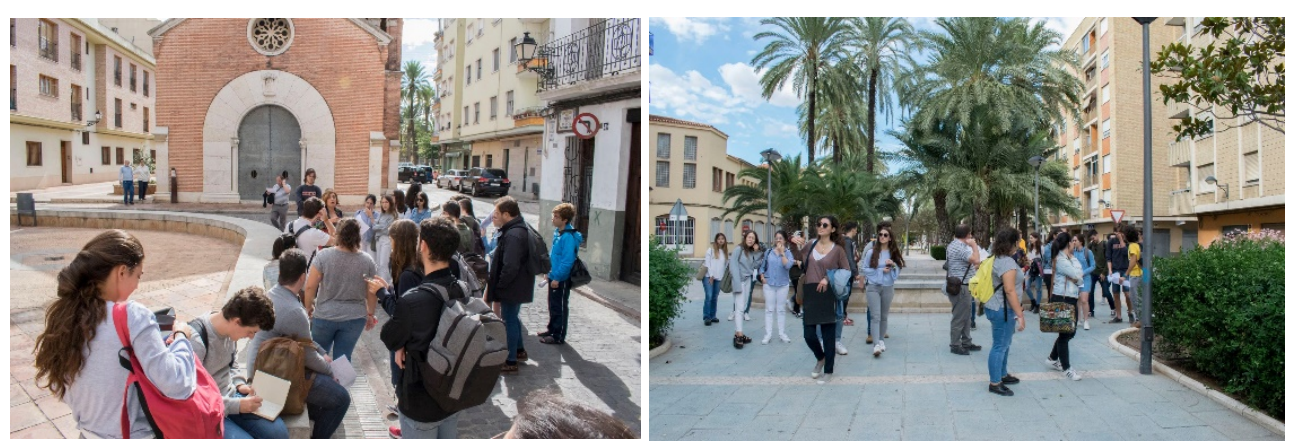

Fig. 2 y 3. Visita y reconocimiento del alumnado al espacio público de Algemesí

Noviembre 2017. El arquitecto municipal acude a la Universidad para impartir una clase magistral acerca de la evolución urbana de Algemesí. Con la toma de datos sobre el municipio y después de la clase impartida por el arquitecto municipal, los alumnos y alumnas inician un trabajo de análisis de los barrios de Algemesí atendiendo a aspectos morfológicos y funcionales, este trabajo se prolonga hasta el mes de diciembre

Diciembre 2017. Entrega del trabajo de análisis del barrio con una exposición pública de los resultados. Se realiza una valoración crítica y se detectan los principales problemas y oportunidades de intervención para la regeneración urbana.

Enero 2018. Partiendo de las áreas de interés seleccionadas anteriormente para la regeneración urbana, se inicia el segundo ejercicio práctico en equipo que consiste en el análisis del espacio público. Se seleccionan parques y rondas como elementos con mayor grado de uso por parte de la ciudadanía y que se encuentran en un estado de deterioro detectable y visible.

Febrero 2018. Los estudiantes realizan una nueva toma de datos, in situ, por medio de observación directa simple y no participante, durante un intervalo horario definido por patrones de uso del espacio público. Detectan los perfiles de usuarios y los problemas que allí se producen. Además en esas visitas, realizan una catalogación de todos los elementos que definen la materialidad de ese espacio (pavimentos, mobiliario urbano, farolas, tipos de juegos de niños etc.), por medio de fotografías y cuadernos de notas observacionales (quién, qué, cuándo, dónde y cómo de la actividad humana).

Durante el mes de febrero se producen las clases magistrales de los especialistas en sociología y botánica. En este momento se introduce la interdisciplinareidad a la asignatura. Por parte de la experta en sociología se aportan las técnicas de investigación social para el acercamiento a la ciudadanía (observación directa, entrevistas abiertas, encuestas, etc.). 
Por parte del experto en botánica se imparte una clase específica de clasificación de especies vegetales en climas mediterráneos, que les será de utilidad para la evaluación de la calidad ambiental de estos espacios.

Marzo 2018. Se entrega el trabajo de análisis del espacio público realizado en equipo y se presenta en exposición pública. En esta fase se reflejan los resultados del trabajo de análisis del espacio público, incluyéndose datos relativos a la materialidad, al uso y a las condiciones de confort del espacio público. A continuación se hace un diagnóstico y una valoración crítica del estado en que se encuentran estos parques.

Se inicia la fase final del trabajo, la realización del proyecto de mejora del espacio público. Además, el alumnado también realiza un ejercicio individual para aprender a procesar datos de la ciudadanía a través de la plataforma web valenciaparcsdebarri.es. Este ejercicio se realiza en Valencia y se complementa con encuestas in situ.

En esta fase se reflejan los resultados del trabajo de análisis del espacio público

Abril 2018. Se continua con el trabajo en equipo y se introducen clases magistrales de expertos en distintas disciplinas de aplicación a la ejecución del proyecto de mejora en el espacio público: iluminación, calidad ambiental, diseño social, intervenciones históricas, etcétera.

Mayo 2018. Al finalizar el mes de mayo, el alumnado entrega sus trabajos finales.

Junio 2018. Evaluación de los trabajos entregados

\section{Resultados de la innovación}

Los resultados de los trabajos docentes son mostrados al Ayuntamiento mediante una exposición en el municipio, abierta a la ciudadanía. Por otro lado, los trabajos serán también expuestos en la Escuela. Dentro del Plan de diffusion también contaremos con la repercussion dentro de la red internacional Desis Lab.

Aunque los resultados de este curso todavía no están finalizados, con la experiencia previa del curso 2016-17 cuyo trabajo se desarrolló en el municipio de Cullera y del año anterior en Valencia, disponemos de resultados en los que se puede valorar la aplicación de esta metodología. (Fig.4 /Fig. 5)
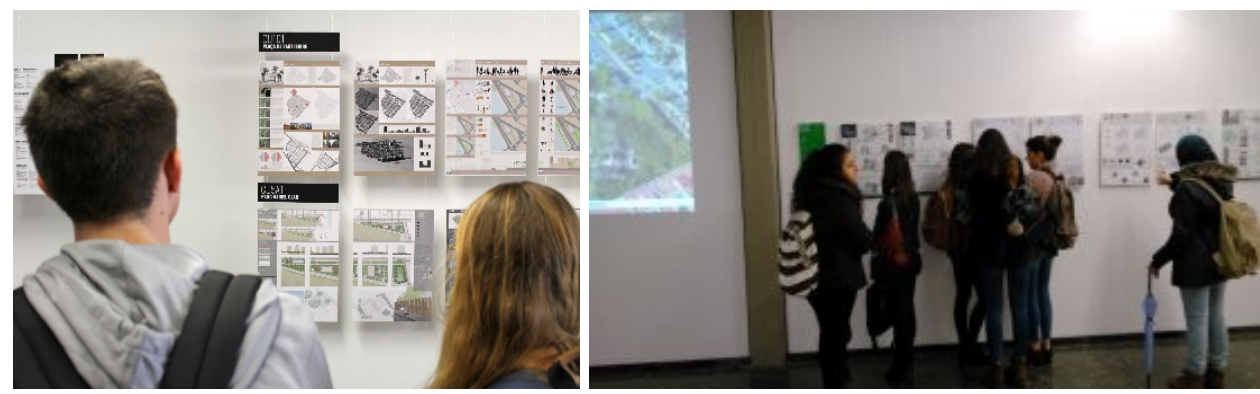

Fig. 4 y 5. Exposición de trabajos docentes en la E.T:S. de Arquitectura 2017

(c) EY-NC-ND 2018, Universitat Politècnica de València

Congreso IN-RED (2018) 
Desde el punto de vista proyectual, la aplicación de una metodología basada en temas reales, en los que se incorpora la participación ciudadana redunda en una mejora en la definición del programa de necesidades del espacio público, mejorando a nivel funcional el Proyecto desarrollado.

Desde el punto de vista de la materialidad, el análisis y la catalogación de los elementos que definen el espacio público, plantea una reflexión de la adecuación o no, de estos elementos, al espacio en el que se integran.

El diseño urbano también mejora sobre todo después de las clases impartidas por distintos especialistas en la material.

\section{Conclusiones}

Los ejercicios demuestran que el trabajo en temas reales, la cercanía a la ciudadanía y a la administración, desarrollan una motivación especial en los alumnos. La incorporación de clases de expertos refuerzan la importancia de la interdisciplinareidad en los proyectos de regeneración urbana.

Por otro lado, el trabajo en equipo de 4 o 5 alumnos integrantes, también ayuda a la toma de decisiones y acerca a la realidad del ejercicio profesional, que en urbanismo, fundamentalmente se desarrolla por medio del trabajo en equipos multidisciplinares.

Esta innovación aporta al alumnado un modo de acercamiento al espacio público que aúna la componente social con la urbana. Plantea una reflexión abierta sobre la funcionalidad y materialidad de los parques de barrio. Desde esta reflexión, surgen los argumentos para afrontar con mayor solvencia los proyectos de regeneración urbana y mejora en los espacios públicos.

Destacamos también la importancia de la difusion de las metodologías docentes activas y los resultados que generan, en este sentido la integración del Proyecto en la red DESIS-Lab aporta repercusión a esta iniciativa docente.

\section{Referencias}

DÍEZ-MEDINA, C., \& MONCLÚS-FRAGA, J. (2017). "Docencia e investigación: exploraciones teórico-proyectuales en torno a la regeneración urbana" $V$ Jornadas sobre innovación docente en arquitectura. Universitat Politècnica de Catalunya. Iniciativa Digital Politècnica. Grup per a la Innovació i la Logística Docent en l'Arquitectura. Disponible < https://upcommons.upc.edu/handle/2117/109609> [Consulta: 20 de marzo 2018.]

GULBAHAR, Y. TINMAZ, H. (2006). "Implementing Project-Based Learning and E-Portfolio Assessment in an Undergraduate Course". Journal of Research on Technology in Education, vol. 38, no3, p. 309-327.

MARTíNEZ, M. (2008). “Aprendizaje servicio y construcción de ciudadanía activa en la universidad: la dimensión social y cívica de los aprendizajes académicos”. En Miquel Martínez (ed) Aprendizaje servicio y responsabilidad social de las universidades. Barcelona: Ministerio de Educación y Ciencia 
Innovación docente y participación ciudadana en proyectos de regeneración urbana. PIME "Desis Lab: Proyecto parques urbanos de barrio"

(MEC), Secretaría General Técnica y Ediciones Octaedro, S.L..; 11-26 < http://www.ucv.ve/uploads/media/Aps y universidad.pdf> [Consulta: 14 de marzo 2018].

ODRIA, A. M. (2007). "Service-learning o aprendizaje-servicio: la apertura de la escuela a la comunidad local como propuesta de educación para la ciudadanía”. Bordón. Revista de pedagogía,Vol. 59, Núm 4, p. 627-640. Disponible https://recyt.fecyt.es/index.php/BORDON/article/view/36336> [Consulta: 14 de marzo 2018]

PORTALES MAÑANOS, A.; PALOMARES FIGUERES, M. T.; ESTEVE SENDRA, M. C.; PASCUAL-SEVA, N. (2015). "La participación social aplicada al aprendizaje en proyectos. Su desarrollo metodológico en asignaturas de diseño urbano y diseño de producto”. 23 Congreso Universitario de Innovación Educativa en las enseñanzas Técnicas. pp. 951 - 965. 